УДК 517.9

\title{
Magnetic Vortices in Ferromagnetic Nanodots
}

\author{
Roman Yu.Rudenko* \\ Anatoly V. Chzhan ${ }^{\dagger}$ \\ Siberian Federal University \\ Svobodny, 79, Krasnoyarsk, 660041 \\ Kirensky Institute of Physics \\ Russian Academy of Sciences SB RAS \\ Akademgorodok, 50/38, Krasnoyarsk, 660036
}

Russia

Received 09.05.2015, received in revised form 10.06.2015, accepted 19.07.2015

Studies in the field of magnetodynamics of vortex structures are reviewed. The prospects for application of ferromagnetic nanodots in high-speed nonvolatile memory are demonstrated. The creation of a static magnetic vortex is described. The available theoretical techniques for describing the motion of a magnetic vortex are discussed. The experimental and theoretical works on the vortex motion in dynamic magnetic fields of the gigahertz and subgigahertz frequency ranges are considered. The issue of the effect of the interaction between nanodots on the dynamic properties of nanodot arrays is raised.

Keywords: nanodots, magnetic films, ferromagnetic resonance.

DOI: 10.17516/1997-1397-2015-8-3-327-339

\section{Introduction}

The first magnetic recording device was developed by Danish engineer Valdemar Poulsen. It was called telegraphone and patented in 1898. The real breakthrough in magnetic recording has happened on May 21 1952, when an IBM 726 tape drive for an IBM 701 computer was announced. In modern digital storage devices ferromagnetic films have found wide application. In the process of digital magnetic recording current flowing in a recording head reverses the magnetic field direction at certain intervals. As a result, under the action of the scattering field of a magnetic head some regions of a moving magnetic carrier are switched. This way of data storage is nonvolatile, i.e., further data storage after switching the cells by a recording head does not require electric power. Magnetic memory is characterized by high ( up to $\sim 10^{12} \mathrm{bit}_{/} \mathrm{inch}^{2}$ ) recording density and it is widely used in data storage devices and hard disc drives (HDD). However, the rate of data exchange in such devices is limited because data recording and reading are performed by the same head.

An alternative is high-speed dynamic random access memory (DRAM) in which a bit of data is stored in the form of capacitor charge. A memory cell is comprised of capacitor and transistor. The capacitor is charged to the highest or lowest voltage (logic 1 or 0 ). The transistor works as a switch which connects the capacitor to a control circuit integrated in the same chip. The control circuit allows reading the state of a capacitor charge or changing it. A drawback of DRAM is

\footnotetext{
*rudenko-roma406@ya.ru

†avchz@mail.ru

(c) Siberian Federal University. All rights reserved
} 
that capacitors need to be periodically recharged. To store the memory contents the capacitor charge is regenerated with an interval of $\sim 2 \mathrm{~ms}$. It is implemented in modern chips with the use of a reading cyclogram over all rows of the storing matrix. Since during memory regeneration the memory access of the central processor periodically stops the average rate of data exchange is reduced.

This problem was solved by the development of static random access memory (SRAM) where a bit of data is stored in the form of a trigger state. This memory is expensive but it has a shorter access time and lower energy consumption as compared with DRAM. Since triggers consist of several logic gates with a very short delay the trigger state is switched very fast. However, this memory has certain drawbacks. Transistors contained in a trigger occupy much larger area on a chip than a dynamic memory cell because a trigger consists of at least two gates with 6-8 transistors. Therefore, the trigger is more expensive than the dynamic memory cell that consists of one transistor and one capacitor. For this reason, despite a relatively low data capacity, SRAM is used in high-speed memory devices in which the data exchange is critical for system performance. In particular, the use of SRAM in cache memory of the central processing unit significantly enhances the computer power.

Low-cost nonvolatile magnetic random access memory (MRAM) with the speed comparable to the speed of SRAM and the chip memory of few Gbyte can be formed from ferromagnetic nanodots with the vortex magnetic structure [1]. The prospect of application of such systems in MRAM is determined by the possibility of simultaneous control of many nanodots in an array and by high stability of memory cells due to weak scattering fields and, consequently, minimum cross-noise.

\section{Main magnetic state of ferromagnets. Magnetic vortex}

Demagnetizing fields make the single-domain state of macroscopic ferromagnets energetically unfavorable. A crystal is divided into several domains when external magnetic field is absent. The domain structure (DS) depends on the magnetic parameters of a sample and on its shape. For instance, in the case of easy-axis anisotropy and far from the edge of a magnet, the equilibrium DS is the structure where neighboring domains are magnetized in antiparallel manner (Fig 1).

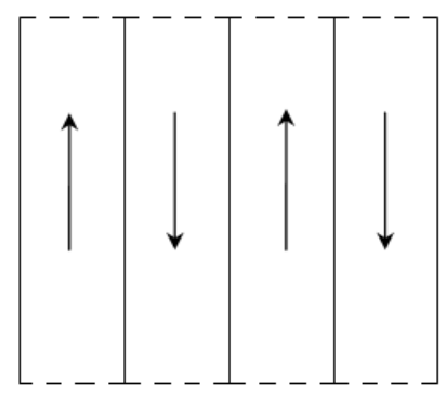

Fig. 1. Distribution of magnetization in a thin film

There is a transition region between domains. It is called the domain wall (DW). It was described first by Landau and Lifshitz [2]. They demonstrated that magnetization in a DW is changed smoothly rather than step-wise in the region of size $\delta$. The region contains a finite number of atomic layers. In 180-degree domains magnetization at the DW center is directed at 
the angle of $90^{\circ}$ to the domain magnetization. In thin magnetic films (TMFs) this rotation can occur in various directions relative to the DW plane under variations of film thickness. This leads to the formation of different types of DWs. The simplest types are Neel and Bloch walls, along with the intermediate type of walls with cross bonds. The occurrence of either wall type is related to the decrease in demagnetizing field caused by the magnetization distribution inside a DW. When film thickness $d<\delta$ then DW magnetization lies in the film plane. This is characteristic property of Neel walls (Fig. 2a). As the thickness is increased Bloch walls with the out-of-plane magnetization become energetically more favorable (Fig. 2b). The configuration illustrated in the figure reduces the energy of demagnetizing field of the wall. The domain wall width is determined from the condition of a balance between the exchange energy and the crystallographic magnetic anisotropy. The exchange energy is decreased with an increase in $\delta$ but the crystallographic magnetic anisotropy is increased with a decrease in $\delta$.

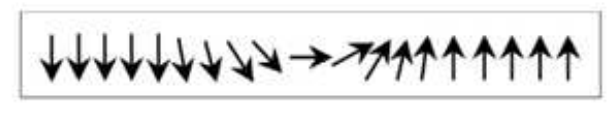

a)

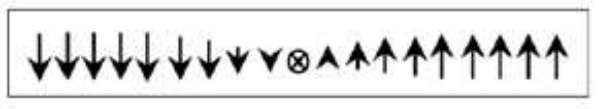

b)

Fig. 2. (a) Neel and (b) Bloch domain walls

In nanosized TMFs called magnetic spots, nanodisks or simply nanospots the magnetic state is mainly determined by the exchange energy $E_{e x}$ and magnetostatic energy $E_{m s}$. Depending on size and shape of a magnet, different states can be implemented from single-domain to multiplevortex structures with the out-of-plane magnetization and formation of the so-called cores. The state formation depends on two factors. The magnetostatic energy tends to decrease the energy at the sample edges with the formation of a non uniform magnetization distribution but the exchange energy tends to arrange spins parallel to each other. In theory, depending on the ratio between $E_{e x}$ and $E_{m s}$, three magnetic states can be implemented in nanodisks: two singledomain states with the in-plane and perpendicular magnetization and a vortex state. In the single-domain state the magnetization direction relative to the film plane is determined by the ratios $R / L_{e x}$ and $L / L_{e x}$, where $L$ is the film thickness, $R$ is the disk radius and $L_{e x}$ is the exchange length of a material. The region of existence of the single-domain and vortex phases can be established with the use of the experimental phase diagram (Fig. 3) [3].

At the center of the magnetic vortex (MV), the exchange energy is high due to the large angle of rotation between neighboring spins. Therefore, in order to minimize this energy the magnetization in the central region goes out of plane and it is arranged perpendicular to the plane. This region of the MV is called core. The magnetic vortex is a three-dimensional structure. Core radius $r_{c}$ is comparable with the exchange length $L_{e x}=\sqrt{A / M_{S}^{2}}$, where $A$ is the exchange constant and $M_{S}$ is the saturation magnetization [4]. For example, in the amorphous Co-P film with $M_{S}=1020 \mathrm{G}$ and $A \approx 10^{-6} \mathrm{erg} / \mathrm{cm}$, we have $r_{c} \approx 10 \mathrm{~nm}$.

In square films the MV arises at the Neel wall crossings (Fig. 4a) with the formation of a core at the center in the form of a Bloch point. In disk-shaped films the MV can occupy the entire spot volume with the core at the center (Fig. 4b). 


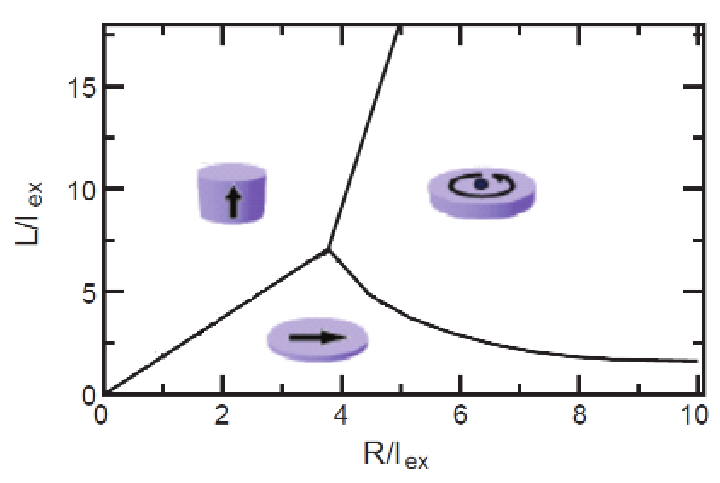

Fig. 3. Phase diagram of the formation of single-domain and vortex states

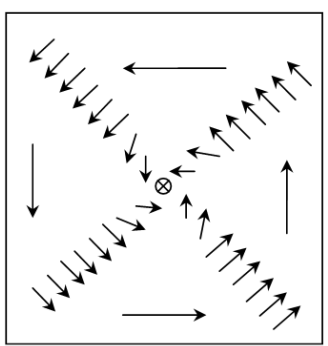

a)

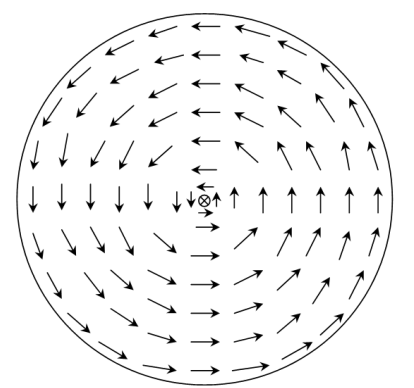

b)

Fig. 4. Configurations of magnetic vortices in square and circular nanodots

The magnetic vortex is characterized by the two parameters: the vortex chirality $q= \pm 1$ which indicates the magnetization direction (clockwise or counterclockwise) and the core polarity $p= \pm 1$ which indicates the direction of magnetization in the core (along the $\mathrm{z}$ axis or opposite to it). The magnetization at the core center is perpendicular to the plane [5,6] (Fig. 5). The bit state in storage devices can be coded using core polarity $p$ and/or vortex chirality $q$ [7] (Fig. 6). This is a serious advantage over the devices where a bit of data is determined only by the two magnetization states.

Polarity of a vortex core was established for the first time in the year 2000 on permalloy samples with the use of a magnetic force microscope [8]. The core polarity was changed by a magnetic field of $15 \mathrm{kOe}$ applied perpendicular to the nanodisk plane.

Since ferromagnetic nanodots with the vortex structure are intended for application in memory cells their dynamic characteristics are of great importance $[9,10]$. In recent years the dynamic properties of ferromagnetic nanodot arrays have been studied both experimentally and theoretically [11-16]. The numerical and analytical calculations were performed with the use of various model functions of magnetization distribution inside the vortex $[6,17,18]$. Because these functions are always well-localized functions the main characteristic of a vortex is its size. Therefore, the results obtained by different authors differ insignificantly.

At the same time, it should be noted that a specific shape of the magnetization distribution function can be important for studying high-frequency (gigahertz and higher) modes. In this 


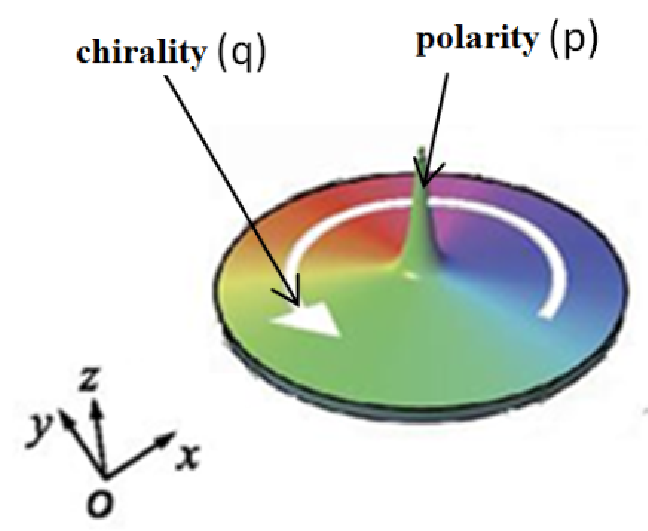

Fig. 5. Vortex chirality and core polarity in the disk-shaped film

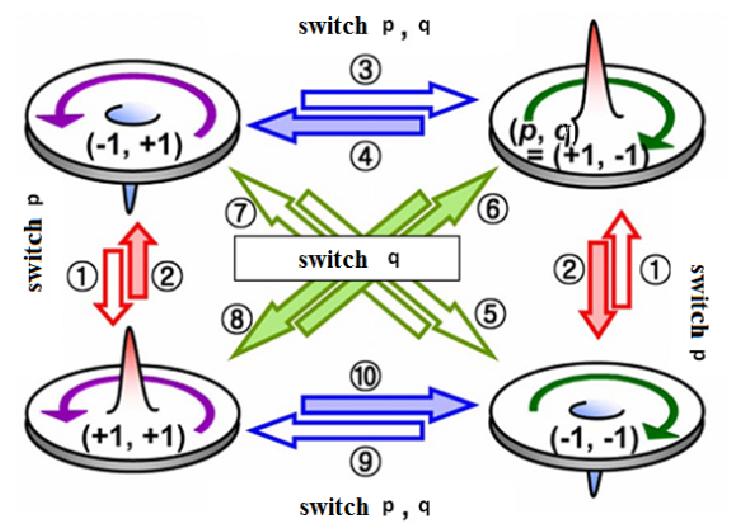

Fig. 6. Information coding with the use of polarity and chirality

case, the magnetization evolution is not a simple gyrotropic motion of the core as a quasi-particle (it would be discussed below) but it is complex spin-wave oscillation [19]. The vortex profiles frequently used in theoretical calculations describe the real situation with insufficient accuracy, especially in the presence of external magnetic field. Magnetic field applied perpendicular to the magnet plane modifies the magnetization distribution in the core. As a result, far from the core the magnetization goes out of the nanodisk plane. This leads to the change in the magnet effective energy, core mass and the gyromagnetic constant. This causes the variation in the frequency of gyrotropic motion [9, 20, 21, 23].

\section{Collective variables technique in the description of the vortex core motion}

In external magnetic field $\mathbf{H}$, a particle with the magnetic moment $\mathbf{m}$ has the precession motion in accordance with the equation

$$
\frac{d \mathbf{m}}{d t}=-\gamma \mathbf{m} \times \mathbf{H}
$$


The motion of magnetization $\mathbf{M}$ in effective field $H_{e f f}$ is generally described by the LandauLifshitz equation [2]:

$$
\frac{d \mathbf{M}}{d t}=-\gamma \mathbf{M} \times \mathbf{H}_{e f f},
$$

where $H_{e f f}=H_{e x}+H_{d}+H_{Z e e m a n}$. This equation is valid for a medium without dissipation and spin-polarized current. In the Landau-Lifshitz equation the effective field is the sum of internal effective fields, including exchange field $H_{e x}$ and demagnetizing field $H_{d}$, and external Zeeman field $H_{Z e e m a n}$ and others. Hilbert added to Eq. (2) the decay constant $\alpha$ that characterizes the rate of energy dissipation caused, e.g., by crystal impurities in a material [24]. Taking into account the Hilbert decay parameter, the Landau-Lifshitz equation takes the form

$$
\frac{d \mathbf{M}}{d t}=-\gamma \mathbf{M} \times \mathbf{H}_{e f f}+\frac{\alpha}{M_{S}} \mathbf{M} \times \frac{d \mathbf{M}}{d t} .
$$

Taking into account also the spin momentum, one can rewrite the Landau-Lifshitz equation in the form [25]:

$$
\frac{d \mathbf{M}}{d t}=-\gamma \mathbf{M} \times \mathbf{H}_{e f f}+\frac{\alpha}{M_{S}} \mathbf{M} \times \frac{d \mathbf{M}}{d t}-\frac{b_{j}}{M_{S}^{2}} \mathbf{M} \times[\mathbf{M} \times(\mathbf{J} \cdot \nabla) \mathbf{M}]-\frac{\xi b_{j}}{M_{S}} \mathbf{M} \times(\mathbf{J} \cdot \nabla) \mathbf{M},
$$

where $b_{j}=\mu_{B} P / e M_{S}\left(1+\xi^{2}\right)$ is the constant of coupling between itinerant spins and magnetization, $\mu_{B}$ is the Bohr magneton, $\mathbf{J}$ is the electric current density, $P$ is the spin polarization of the current and $\xi$ is the ratio of exchange relaxation time $\tau_{e x}$ to spin relaxation time $\tau_{s f}$.

It is well-known that the motion of the magnetic vortex core is similar to the Larmor motion of a charged particle around magnetic lines. This was theoretically justified for the first time by Thiele [26]. The vortex magnetization state can be described using generalized coordinates $\{\mathbf{X}, \mathbf{v}\}$ (core coordinate and velocity). Then, the Landau-Lifshitz equation becames

$$
\mathbf{G} \times \frac{d \mathbf{X}}{d t}+\mathbf{D} \frac{d \mathbf{X}}{d t}-\frac{\partial W(\mathbf{X})}{\partial \mathbf{X}}=0
$$

Here $\mathbf{X}(x, y)$ is the core center position. The gyromagnetic force is proportional to the gyromagnetic vector $\mathbf{G}=G \mathbf{z}$, where $G=2 \pi q p L M_{S} / \gamma$ is the gyromagnetic constant and $\mathbf{D}$ is the dissipation parameter. The solution of Eq. (5) is the core trajectory in the form of a convergent helix.

It should be noted that Eq. (5) is approximate and yields the solution for the gyrotropic motion of the vortex core in the low-frequency $(<1 \mathrm{GHz})$ range. However, in recent theoretical studies [27-30] the modified Thiele equation was analyzed with regard to the terms of higherorder derivatives of the coordinate with respet to time:

$$
\mathbf{G}_{3} \times \ddot{\mathbf{v}}+\widehat{\mu}_{*} \dot{\mathbf{v}}+\mathbf{G} \times \mathbf{v}+\nabla W+\widehat{D} \mathbf{v}=0 .
$$

Here $\mathbf{v}$ is the core velocity, $\widehat{\mu}_{*}$ is the tensor of the effective mass of the magnetic core, $\mathbf{G}_{3}$ is the third-order gyromagnetic vector and $\widehat{D}$ is the decay tensor. The solution of Eq. (6) is a complex trajectory which is similar to a convergent helix with superimposed high-frequency librations. The libration frequencies are of few gigahertz and they depend on the core effective mass. As a rule, the libration-related effects are ignored in studies. Meanwhile, the libration frequency variation allows us to determine the core mass in experiments.

In applied AC magnetic field with the frequency similar to that of the core motion the core starts rotating around the nanodot center. It is the mode when the core polarity (bit state) variation becomes energetically more favorable. 


\section{Controlling the magnetic vortex state}

There is still a lack of a reliable method for controlling chirality and polarity of the MV. Here we consider a few ways of controlling the MV state that are discussed in the literature.

Short magnetic-field pulses. Switching of vortex core polarity $p$ in weak fields was experimentally investigated [11]. The vortex was excited in nanodots by the in-plane sinusoidal magnetic field, which led to the gyrotropic motion of the vortex core relative to the equilibrium position.

The vortex core excitation dynamics was studied [7]. The helical motion of the vortex at high-power field was observed. It was established that a short pulse causes the initial linear acceleration of the core in the magnet plane. Then controlled gyrotropic motion follows. Chirality is independent of the initial acceleration direction or the core rotation direction. In addition, it was demonstrated that sub-nanosecond pulses of the in-plane magnetic field, which are shorter than the relaxation time, initially displace the core collinearly to the applied field.

To get an idea of the properties of mesoscopic magnetic structures used in magnetoelectronic devices it is necessary to study the magnetization dynamics in the above-mentioned time scales.

Spin-polarized current control. The gyrotropic linear and nonlinear motion of the magnetic vortex in magnetically soft cylindrical nanodots was studied [22] with the use of micromagnetic simulation and the Thiele equation of motion [26]. It was established that orientation of the magnetic vortex can be dynamically switched using not only magnetic-field oscillations with small amplitudes but also AC currents [31-33]. Electric-field switching of polarity $p$ of the vortex core is implemented through the dynamic excitation at the frequency equal to the vortex resonance frequency. The core is switched by strong dynamic field which is locally induced by high-speed (hundreds of $\mathrm{m} / \mathrm{s}$ ) rotation of the core. In addition, the dynamic switching is closely related to the gyrotropic motion of the vortex core.

This is an essential step in the way to implementate of nanodot magnetic memory. The fundamental understanding of the vortex core motion is of great importance especially in the case of the resonance conditions.

Observation of low-frequency modes. Magnetic nanodots in the vortex ground state are characterized by two qualitatively different spin excitation modes: low-frequency gyrotropic $[19,20]$ and high-frequency radial and azimuth modes [12, 34]. Although the resonant excitation of the gyrotropic motion has been intensively studied [12-46] many dynamic properties of the vortex structure of nanodots and their arrays remain poorly understood. In particular, the mechanism of creating the vortex-antivortex pair is still unclear. In addition, it is interesting to clarify the character of the nonlinear magnetic vortex oscillation and the effect of the magnetostatic interaction between nanodots in array on the oscillation modes [47-51].

There was an attempt to describe the vortex core motion through the superposition of eigenmodes and the asymmetric resonance effect [21, 22]. Micromagnetic numerical simulation of the vortex dynamics on the basis of Landau-Lifshitz-Hilbert equation was used (4). Analytical calculations based on Thiele equation of motion (5) were performed.

Experimental observation of the low-frequency modes corresponding to the vortex core rotation near the equilibrium position was reported [52]. It was demonstrated that the magnetic field applied in the sample plane weakly affects the resonance frequency, shifting it within $5 \%$. This effect becomes noticeable when field is close to the vortex annihilation field.

The dynamic properties of the core can be successfully studied using a coplanar waveguide in the reflection mode [53]. However, this technique is inapplicable for studying the ferromagnetic 
resonance in a wide frequency range.

Observation of high-frequency azimuth modes. Understanding of the high-frequency excitation of modes (spin waves) allow us to develop a technique for controlling the magnetic-fielddriven vortex switching $[54,55]$. High-frequency azimuth modes determine the distortion of the vortex core upon switching the vortex in perpendicular magnetic field [56]. The eigenfrequency of magnetization oscillations in a nanodot also depend on the nanodot shape, all other factors being equal.

There are various experimental techniques for observation of high-frequency azimuth modes $[35,36,52]$. A series of experiments on measuring the excited spin-wave azimuth modes was carried out and it was established that the eigenfrequencies are much lower than the frequencies obtained by computer simulation [35].

The measurements of azimuth and radial spin-wave modes at the broadband ferromagnetic resonance in zero magnetic field for circular permalloy nanodisks with the vortex magnetization structure were reported [37]. These experimental results agree well with the results of simulation presented in [36].

The experimental and theoretical results of an investigation into a set of high-frequency (gigahertz) modes was presented in [38]. The dependences $H(\omega)$ were obtained and the effect of the disk size on the high-frequency spectrum was considered. Studies of the high-frequency spin-wave excitation modes in ferromagnetic nanodisks in the vortex state are of interest to both fundamental research and applications.

Observation of the vortex structure. The vortex structure can be observed and studied with the use of different experimental methods. Magneto-optical Kerr effect microscopy allows the detail analyzis of the spin-wave excitations and their frequencies, intensity and spatial distribution $[12,34,57]$. The high-frequency coupled spin waves in the vortex state were experimentally observed for the first time with the use of Brillouin light scattering (BLS) spectroscopy, in which reflected light is scattered on acoustic hypersonic wave generated by an excited nanodot due to magnetostriction [39]. The high-frequency spin waves were studied in detail and eigenfrequencies in the subgigahertz range were not revealed [40, 41].

The results of calculations of radial spin-wave eigenfrequencies and profiles for different modes in the vortex state of ferromagnetic nanodisks were presented in [43]. Radial high-order spin waves in the vortex state were found using BLS spectroscopy which allows observing the intensity distribution (sites and antinodes) for radial modes up to the fifth order. The higher modes cannot be observed because of the limited spatial resolution of the technique.

Double vortex state. An understanding of the dynamic interaction between vortex cores and between magnetic nanodot arrays is very important. The coupling between rotations of vortices in double disk structures was considered in [44-46]. The coupling between cores was discussed in [45]. It was mentioned that the most significant consequences of this interaction are the reduction of the resonance frequency of coupled modes and asymmetry of the spectrum.

A series of experiments on the magnetization dynamics in circular permalloy nanodots with separated magnetic vortices coupled with each other by DWs (double vortex state) was reported in [58].

Although the nature of excited states of DWs and excited vortex states is well-studied [12, 19, $58,59,60]$, the mechanism of spin-wave excitation in magnetic structures with vortices and DWs calls for further investigations. The evolution of broadband dynamic characteristics of a nanodot was investigated in the experiments as a function of the slowly varying in-plane magnetic field. 


\section{Conclusion}

The processes that occur in ferromagnetic nanodots attract much attention all over the world, whereas the technique for controlling vortex chirality and polarity is still underdeveloped. Thus, the actual problems to be solved are

(1) development of methods for controlling vortex chirality and core polarity, study of highfrequency regimes of the vortex magnetization motion and ferromagnetic resonance;

(2) experimental studies of the high-frequency modes of the magnetization oscillations in magnets with the vortex structure;

(3) experimental and theoretical study of the effect of the interdisk interaction in nanodot arrays on the dynamic characteristics of magnetization.

\section{References}

[1] K.Bussmann, G.A.Prinz, S.-F.Cheng, D.Wang, Switching of vertical giant magnetoresistance devices by current through the device, Appl. Phys. Lett., 75(1999), 2476.

[2] L.D.Landau, E.M.Lifshitz, On the theory of the dispersion of magnetic permeability in ferromagnetic bodies, Phys. Z. Sowjetunion, 8(1935), 153.

[3] W.Scholz, K.Yu.Guslienko, V.Novosad, D.Suess, T.Schrefl, Micromagnetic simulation of the pinning and depinning process in permanent magnets, JMMM, 266 (2003), 155-163.

[4] A.Wachowiak et al., Direct Observation of Internal Spin Structure of Magnetic Vortex Cores, Science, 298(2002), 577 .

[5] J.Raabe, R.Pulwey, R.Sattler, T.Schweinbock, J.Zweck, D.Weiss, Magnetization pattern of ferromagnetic nanodisks, J. Appl. Phys., 88(2000), 4437.

[6] K.Y.Guslienko, K.-S.Lee, S.-K.Kim, Dynamic Origin of Vortex Core Switching in Soft Magnetic Nanodots, Phys. Rev. Lett., 100(2008), 027203.

[7] Y.-S.Choi, M.-W.Yoo, K.-S.Lee, Y.-S.Yu, H.Jung, S.-K.Kim, Out-of-plane current controlled switching of the fourfold degenerate state of a magnetic vortex in soft magnetic nanodots, Appl. Phys. Lett., 96(2010), 072507.

[8] T.Shinjo, T.Okuno, R.Hassdorf, K.Shigeto, T.Ono, Magnetic Vortex Core Observation in Circular Dots of Permalloy, Science, 289(2000), 930.

[9] B.Pigeau, G.De Loubens, O.Klein, A.Riegler, F.Lochner, G.Schmidt, L.W.Molenkamp, V.S.Tiberkevich, A.N.Slavin, Magnetic Vortex Core Observation in Circular Dots of Permalloy, Appl. Phys. Lett., 96(2010), 132506.

[10] K.Y.Guslienko, V.Novosad, Vortex state stability in soft magnetic cylindrical nanodots, $J$. Appl. Phys., 96(2004), 4451.

[11] B. Van Waeyenberge, A.Puzic, H.Stoll, K.W.Chou, T.Tyliszczak, R.Hertel, M.Faehnle, H.Brueckl, K.Rott, G.Reiss, I.Neudecker, D.Weiss, C.H.Back, G.Schuetz, Magnetic vortex core reversal by excitation with short bursts of an alternating field, Nature (London), 444(2006), 461. 
[12] M.Buess, R.Hollinger, T.Haug, K.Perzlmaier, U.Krey, D.Pescia, M.R.Scheinfein, D.Weiss, C.H.Back, Fourier Transform Imaging of Spin Vortex Eigenmodes, Phys. Rev. Lett., 93(2004), 077207.

[13] K.Perzlmaier, M.Buess, C.H.Back, V.E.Demidov, B.Hillebrands, S.O.Demokritov, SpinWave Eigenmodes of Permalloy Squares with a Closure Domain Structure, Phys. Rev. Lett., 94(2005), 057202.

[14] H.Schultheiss, S.Schafer, P.Candeloro, B.Leven, B.Hillebrands, A.N.Slavin, Observation of Coherence and Partial Decoherence of Quantized Spin Waves in Nanoscaled Magnetic Ring Structures, Phys. Rev. Lett., 100(2008), 047204.

[15] S.Kasai, Y.Nakatani, K.Kobayashi, H.Kohno, T.Ono, Current-Driven Resonant Excitation of Magnetic Vortices, Phys. Rev. Lett., 97(2006), 107204.

[16] K.Vogt, H.Schultheiss, S.J.Hermsdorfer, P.Pirro, A.A.Serga, B.Hillebrands, All-optical detection of phase fronts of propagating spin waves in a Ni81Fe19 microstripe, Appl. Phys. Lett., 95(2009), 182508.

[17] Yu.B.Grebenschikov, N.A.Usov, K.Yu.Guslienko, Interaction Effects in a Two-Dimensional Array of Ferromagnetic Dots, Materials Science Forum, 373-376(2001), 149-152.

[18] V.S. Prokopenko, P.D.Kim, V.A.Orlov, B.V.Vasiliev, D.K.Vovk, S.E.Zatsepilin, R. Yu.Rudenko, Lorentz Microscopy of Permalloy Film Microdots, Journal of SFU. Mathematics and Physics, 6(2)(2013), 262-269.

[19] K.Yu.Guslienko, B.A.Ivanov, V.Novosad, Y.Otani, H.Shima, K.Fukamichi, Eigenfrequencies of vortex state excitations in magnetic submicron-size disks, Journal of Applied Physics, 91(2002), 8037.

[20] V.Novosad, F.Y.Fradin, P.E.Roy, K.S.Buchanan, K.Y.Guslienko, and S.D.Bader, Magnetic vortex resonance in patterned ferromagnetic dots, Phys. Rev. B, $72(2005), 244455$.

[21] Ki-Suk Lee, S.-K. Kim, Gyrotropic linear and nonlinear motions of a magnetic vortex in soft magnetic nanodots, Apll. Phys. Lett., 91(2007), 132511.

[22] Ki-Suk Lee, S.-K.Kim, Two circular-rotational eigenmodes and their giant resonance asymmetry in vortex gyrotropic motions in soft magnetic nanodots, Phys. Rev. B, 78(2008), 014405 .

[23] O.Klein, G.deLoubens, V.V.Naletov, F.Boust, T.Guillet, H.Hurdequint, A.Leksikov, A.N.Slavin, V.S.Tiberkevich, N.Vukadinovic, Ferromagnetic resonance force spectroscopy of individual submicron-size samples, Phys. Rev. B, 78(2008), 144410.

[24] T.L.Gilbert, A phenomenological theory of damping in ferromagnetic materials, IEEE Trans. Magn., 40(2004), 9464.

[25] S.Zhang, Z.Li, Roles of nonequilibrium conduction electrons on the magnetization dynamics of ferromagnets, Phys. Rev. Lett., 93(2004), 127204.

[26] A.A.Thiele, Steady-State Motion of Magnetic Domains, Phys. Rev. Lett., 30(1973), 230. 
[27] F.G.Mertens, A.R.Bishop, Dynamics of Vortex in Two-Dimentional Magnets, http://arxiv.org/abs/cond-mat/9903037v1.

[28] B.A.Ivanov, G.G.Avanesyan, A.V.Khvalkovskiy, N.E.Kulagin, C.E.Zaspel, K.A.Zvezdin, Non-Newtonian dynamics of the fast motion of a magnetic vortex, Journal of Experimental and Theoretical Physics Letters, 91(2010), no. 4, 178-182.

[29] S.S.Cherepov, B.C.Koop, V.Korenivski, D.C.Worledge, A.Yu.Galkin, R.S.Khymyn, B.A.Ivanov, Core-Core Dynamics in Spin Vortex Pairs, Phys. Rev. Lett., 109(2012), 097204.

[30] P.D.Kim, V.A.Orlov, V.S.Prokopenko, S.S.Zamai, V.Ya.Prints, R.Yu.Rudenko, T.V.Rudenko, On the Low-Frequency Resonance of Magnetic Vortices in Micro- and Nanodots, Physics of the Solid State, 57(2015), 30--37.

[31] K.Yamada, S.Kasai, Y.Nakatani, K.Kobayashi, H.Kohno, A.Thiaville, T.Ono, Electrical switching of the vortex core in amagnetic disk, Nat. Mater., 6(2007), 269.

[32] R.P.Cowburn, Change of direction, Nature Materials, 6(2007), 255.

[33] S.-K. Kim, Y.-S. Choi, K.-S. Lee, K.Y.Guslienko, D.-E. Jeong, Electric-current-driven vortex-core reversal in soft magnetic nanodots, Appl. Phys. Lett., 91(2007), 082506.

[34] J.P.Park, P.Eames, D.M.Engebretson, J.Berezovsky, P.A.Crowell, Imaging of spin dynamics in closure domain and vortex structures, Phys. Rev. B, 67(2003), 020403R.

[35] J.P.Park, P.A.Crowell, Interactions of Spin Waves with a Magnetic Vortex, Phys.Rev.Lett., 95(2005), 167201.

[36] X.Zhu, V.Metlushko, P.Grutter, M.R.Freeman, Broadband spin dynamics of the magnetic vortex state: Effect of the pulsed field direction, Phys.Rev.B, 71(2005), 180408.

[37] A.A.Awad, K.Y.Guslienko, J.F.Sierra, G.N.Kakazei, V.Metlushko, F.G.Aliev, Precise probing spin wave mode frequencies in the vortex state of circular magnetic dots, Appl. Phys. Lett., 96(2010), 012503.

[38] V.Castel, J.Ben Youssef, F.Boust, R.Weil, B.Pigeau, G.de Loubens, V.V.Naletov, O.Klein, N.Vukadinovic, Perpendicular ferromagnetic resonance in soft cylindrical elements: Vortex and saturated states, Phys. Rev. B, 85(2012), 184419.

[39] V.Novosad, M.Grimsditch, K.Y.Guslienko, P.Vavassori, Y.Otani, S.D.Bader, Spin excitations of magnetic vortices in ferromagnetic nanodots, Phys. Rev. B, 66 (2002),052407.

[40] L.Giovannini, F.Montoncello, F.Nizzoli, G.Gubbiotti, G.Carlotti, T.Okuno, T.Shinjo, M.Grimsditch, Spin excitations of nanometric cylindrical dots in vortex and saturated magnetic states, Phys. Rev. B, 70(2004), 172404.

[41] K.Y.Guslienko, W.Scholz, R.W.Chantrell, V.Novosad, Vortex-state oscillations in soft magnetic cylindrical dots, Phys. Rev. B, 71(2005), 144407.

[42] B.A.Ivanov, C.E.Zaspel, Magnon modes for thin circular vortex-state magnetic dots, Phys. Rev. Lett., 94(2005), 027205. 
[43] K.Vogt, O.Sukhostavets, H.Schultheiss, B.Obry, P.Pirro, A.A.Serga, T.Sebastian, J.Gonzalez, K.Y.Guslienko, B.Hillebrands, Optical detection of vortex spin-wave eigenmodes in microstructured ferromagnetic disks, Phys. Rev. B, 84(2011), 174401.

[44] S.Jain, H.Schultheiss, O.Heinonen, F.Y.Fradin, J.E.Pearson, S.D.Bader, V.Novosad, Coupled vortex oscillations in mesoscale ferromagnetic double-disk structures, Phys. Rev. B, 86(2012), 214418.

[45] M.Hubber, J.Zweck, D.Weiss, Experimental observation of switching in ferromagnetic nanoscale double disks, Phys. Rev. B, 77(2008), 054407.

[46] Y.Liu, Y.Hu, A.Du, Vortex dynamics mediated by exchange coupling in permalloy double disks, J. Magn. Magn. Mater., 324(2012), 792.

[47] Y.Liu, Zh.Hou, S.Gliga, R.Hertel, Influenca of the dynamic dipolar interaction on the curentinduced core switch in vortex pairs, Phys. Rev. B, 79(2009), 104435.

[48] J.Shibata, K.Shigeto, Yo.Otani, Dynamics of magnetostatically coupled vortices in magnetic nanodisks, Phys. Rev. B, 67(2003), 224404.

[49] S.Jain, V.Novosad, F.Y.Fradin, J.E.Pearson, V.Tiberkevich, A.N.Slavin, S.D.Bader, From chaos to selective ordering of vortex cores in interacting mesomagnets, NATURE COMMUNICATIONS, 3(2011), 1330.

[50] D.-S. Han, A.Vogel, H.Jung, Ki-Suk Lee, M.Weigand, H.Stoll, G.Schutz, P.Fischer, G.Meier, S.-K. Kim, Wave modes of collective vortex gyration in dipolar-coupled-dot-array magnonic crystals, SCIENTIFIC REPORTS, 3(2013), 2262.

[51] P.D.Kim, V.A.Orlov, R.Yu.Rudenko, V.S.Prokopenko, I.N.Orlova, S.S.Zamai, Collective dynamics of magnetic vortices in an array of interacting nanodots, JETP Letters, 101(2015), 620 .

[52] F.Hoffmann, G.Woltersdorf, K.Perzlmaier, A.N.Slavin, V.S.Tiberkevich, A.Bischof, D.Weiss, C.H.Back, Mode degeneracy due to vortex core removal in magnetic disks, Phys. Rev. B, 76(2007), 014416.

[53] V.P.Denysenkov, A.M.Grishin, Reliable low-power control of ultrafast vortex-core switching with the selectivity in an array of vortex states by in-plane circular-rotational magnetic fields and spin-polarized currents, Rev. Sci. Instrum., 74(2003), 3400.

[54] S.D.Bader, Colloquium: Opportunities in nanomagnetism, Rev. Mod. Phys., 78(2006), 1.

[55] V.S.Pribiag, I.N.Krivorotov, G.D.Fuchs, P.M.Braganca, O.Ozatay, J.C.Sankey, D.C.Ralph, R.A.Buhrman, Magnetic vortex oscillator driven by d.c. spin-polarized current, Nat.Phys., 3(2007), 498.

[56] G.de Loubens, A.Riegler, B.Pigeau, F.Lochner, F.Boust, K.Y.Guslienko, H.Hurdequint, L.W.Molenkamp, G.Schmidt, A.N.Slavin, V.S.Tiberkevich, N.Vukadinovic, O.Klein, Bistability of vortex core dynamics in a single perpendicularly magnetized nano-disk, Phys. Rev. Lett., 102(2009), 177602. 
[57] M.Buess, T.P.J.Knowles, R.Hollinger, T.Haug, U.Krey, D.Weiss, D.Pescia, M.R.Scheinfein, C.H.Back, Excitations with negative dispersion in a spin vortex, Phys. Rev. B, 71(2005), 104415.

[58] L.Thomas, M.Hayashi, X.Jiang, R.Moriya, C.Rettner, S.S.P.Parkin, Oscillatory dependence of current-driven magnetic domain wall motion on current pulse length, Nature, 443(2006), 197.

[59] E.Saitoh, H.Miyajima, T.Yamaoka, G.Tatara, Current-induced resonance and mass determination of a single magnetic domain wall, Nature, 432(2004), 203.

[60] D.Bedau, M.Klaui, S.Krzyk, U.Rudiger, G.Faini, L.Vila, Detection of Current-Induced Resonance of Geometrically Confined Domain Walls, Phys. Rev. Lett., 99(2007), 146601.

\section{Магнитные вихри в ферромагнитных наноточках}

Роман Ю. Руденко

Анатолий В. Чжан

Обзор посвящен исследованиям в области магнитодинамики вихревых структур. В нем раскрывается перспектива использования ферромагнитных наноточек в создании быстродействующей энергонезависимой памяти. Объясняется механизм зарождения статического магнитного вихря. Показаны известные теоретические методы описания движения магнитного вихря. Проведен обзор экспериментальных и теоретических работ, посвященных движению вихря в динамических магнитных полях гигагерцового и субгигагерцового диапазона частот. Поднимается вопрос о влиянии междискового взаимодействия на динамические свойства массивов наноточек.

Ключевые слова: наноточки, магнитные пленки, ферромагнитный резонанс. 